(C)2019 American Physical Society. Access to this work was provided by the University of

Maryland, Baltimore County (UMBC) ScholarWorks@UMBC digital repository on the Maryland Shared Open Access (MD-SOAR) platform.

Please provide feedback

Please support the ScholarWorks@UMBC repository by emailing scholarworks-group@umbc.edu and telling us what having access to this work means to you and why it's important to you. Thank you. 


\title{
Nonlinear topological transitions over a metasurface
}

\author{
G. D’Aguanno ${ }^{\circ},{ }^{1,2, *}$ Y. Hadad $\odot{ }^{3}$ D. A. Smirnova, ${ }^{4}$ X. Ni, ${ }^{4,5,6}$ A. B. Khanikaev, ${ }^{4,5}$ and A. Alù ${ }^{1,4,5,6, \dagger}$ \\ ${ }^{1}$ Department of Electrical and Computer Engineering, The University of Texas at Austin, Austin, Texas 78712, USA \\ ${ }^{2}$ Department of Computer Science and Electrical Engineering, University of Maryland, 1000 Hilltop Circle, Baltimore, Maryland 21250, USA \\ ${ }^{3}$ School of Electrical Engineering, Tel-Aviv University, Tel-Aviv, 69978, Israel \\ ${ }^{4}$ Department of Electrical Engineering, Grove School of Engineering, The City College of the City University of New York, \\ 140th Street and Convent Avenue, New York, New York 10031, USA \\ ${ }^{5}$ Graduate Center of the City University of New York, New York, New York 10016, USA \\ ${ }^{6}$ Photonics Initiative, Advanced Science Research Center, City University of New York, New York, New York 10031, USA
}

(Received 19 August 2019; published 19 December 2019)

\begin{abstract}
We demonstrate a self-induced topological transition and the formation of topologically protected edge states in a two-dimensional array of coupled resonant cavities placed on a Kagome lattice with saturable nonlinearity introduced in the interconnecting bonds. The dynamic response of the structure is shown to be governed by an intensity-dependent band topology. Our findings uncover prospects for generating and controlling topologically nontrivial edge modes in nonlinear metasurfaces, and delineate a roadmap towards self-reconfigurable robust signal transport in various dynamic systems, ranging from acoustics to electronics and optics.
\end{abstract}

DOI: 10.1103/PhysRevB.100.214310

\section{INTRODUCTION}

Topological insulators, the recently discovered exotic states of matter, were originally proposed and studied in the context of condensed matter physics. In the bulk, these materials behave as conventional insulators, with electrons subject to a bulk band gap, while, on the surface of a sample they support the conduction of electrons that are not scattered or dissipated regardless of the presence of surface impurities or how the surface is cut or distorted. This is a consequence of a topological-invariance mechanism, and thereby the effect is termed topological protection [1-10]. In photonics, similar concepts have been subsequently explored to design, for example, waveguides that allow light to flow around large defects and other imperfections without backreflection [11,12]. Photonic protected states have been lately realized, among others, in a two-dimensional array of coupled optical-ring resonators [13], in photonic crystals [14], and in metamaterials [15]. Other studies have also extended these concepts to the acoustic and mechanical realms [16-20].

One of the simplest systems that give rise to topological effects is the one-dimensional (1D) Su-Schrieffer-Heeger (SSH) model, which describes electrons hopping on a 1D chain with staggered hopping amplitudes. This model has been initially used to describe the spontaneous dimerization of polyacetylene [21] and, owing to its relative simplicity, it has been extensively investigated in connection with many interesting phenomena, such as fractional charge, nontrivial edge states, and self-induced nonlinear transitions [22-30]. In particular, it has been predicted [28] and experimentally demonstrated [29] that the 1D SSH model with alternating, linear and nonlinear bonds gives rise to self-induced topo-

\footnotetext{
*giusdag@umbc.edu

†To whom correspondence should be addressed: aalu@gc.cuny.edu
}

logical transitions with exceptional edge-states that decay to a nonzero plateau level. Topologically protected moving solitons have also been semianalytically predicted and their unprecedented immunity against either local or global defects has been numerically studied in the framework of the 1D SSH model [30] and for helical waveguide arrays [31]. In the nonlinear acoustic domain, dynamically tunable metasurfaces have been studied in Ref. [32], amplitude-dependent topological edge states in Ref. [33], and topological transitions in a 1D, nonlinear, spring-mass chain, similar to the SSH model, in Ref. [34]. In this paper we show that nonlinear metasurfaces can support topological transitions and the generation of protected edge states of pure nonlinear origin. We consider a two-dimensional (2D) metasurface made of coupled resonant cavities placed on a Kagome lattice when a saturable nonlinearity in the interconnections is introduced. These results are an important milestone towards the realization of optical, electronic, and acoustic self-reconfigurable and passive wave systems that are immune to disorder effects, opening pathways towards both fundamental and technological research in the area of topological research based on nonlinearities.

\section{MODEL AND RESULTS}

\section{A. Discrete tight-binding model of the nonlinear Kagome lattice}

An array of coupled cavities arranged in a Kagome lattice [35-37], as sketched schematically in Fig. 1(a), can be considered, to some extent, as the 2D analog of the 1D SSH model. The unit cell constitutes of a trimer composed of three identical resonators, each with resonance frequency $\omega_{0}$. The intracell and intercell coupling coefficients, both with dimension of frequency, are denoted by $\kappa$ and $v$, respectively. Two principal axes of the lattice are chosen along $\hat{x}$ and $\hat{s}=(1 / 2) \hat{x}+(\sqrt{3} / 2) \hat{y}$. We associate each resonator in the lattice with a modal amplitude $a_{m, n}^{(j)}$, where $(m, n)$ are the 
(a)

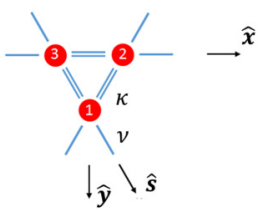

(c)

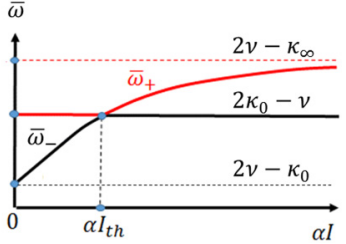

(b)

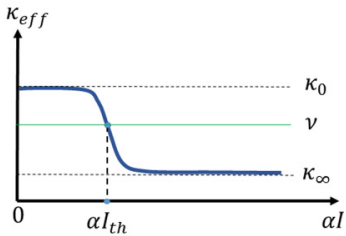

(d)

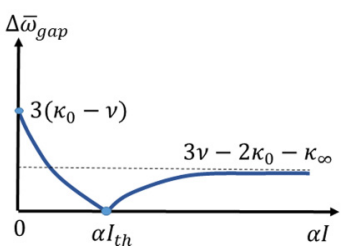

FIG. 1. (a) Elementary cell of the Kagome lattice consisting of three interconnected resonators (trimer) with intracell coupling $\kappa$ and intercell coupling $v$. (b) Effective coupling coefficient vs intensity $\alpha I$. (c) Evolution of the upper and lower band at the Dirac points vs $\alpha I$. (d) Width of the bandgap ( $\left.\Delta \bar{\omega}_{\text {gap }}\right)$ at the Dirac points vs $\alpha I$. The gap closes at $I=I_{\text {th }}$.

integer indices of the specific unit cell, and $j=1,2,3$ is the number of the resonator within the $(m, n)$ 's trimer. The lattice vector is thus defined by $\boldsymbol{r}_{m, n}=m a \hat{s}+n a \hat{x}$, where $a$ is the lattice constant. The time evolution of the coupled resonators is described by the set of nonlinear differential equations

$$
\begin{aligned}
i \frac{d}{d t}\left(\begin{array}{l}
a_{m, n}^{(1)} \\
a_{m, n}^{(2)} \\
a_{m, n}^{(3)}
\end{array}\right)= & \left(\begin{array}{ccc}
\omega_{0} & \kappa_{m, n}^{(2,1)} & \kappa_{m, n}^{(3,1)} \\
\kappa_{m, n}^{(1,2)} & \omega_{0} & \kappa_{m, n}^{(3,2)} \\
\kappa_{m, n}^{(3,1)} & \kappa_{m, n}^{(2,3)} & \omega_{0}
\end{array}\right)\left(\begin{array}{l}
a_{m, n}^{(1)} \\
a_{m, n}^{(2)} \\
a_{m, n}^{(3)}
\end{array}\right) \\
& +v\left(\begin{array}{c}
a_{m+1, n-1}^{(2)}+a_{m+1, n}^{(3)} \\
a_{m-1, n+1}^{(1)}+a_{m, n+1}^{(3)} \\
a_{m-1, n}^{(1)}+a_{m, n-1}^{(2)}
\end{array}\right) .
\end{aligned}
$$

Here, we assume that the intercell coupling $v$ is linear, while the nonlinear response is introduced in the intracell bonds. The intensity-dependent intracell coupling coefficient is taken in the form

$$
\kappa_{m . n}^{(i, j)}=\kappa_{m, n}^{(j, i)}=\kappa_{\infty}+\frac{\kappa_{0}-\kappa_{\infty}}{1+\alpha\left|a_{m, n}^{(j)}-a_{m, n}^{(i)}\right|^{2}}
$$

implying a saturable nonlinearity with the nonlinear parameter $\alpha>0$ in units of inverse of power per unit area. This nonlinearity models well the type of nonlinear coupling achievable with back-to-back varactors or related electronic or photonic implementations [29,30]. We emphasize that, as opposed to the common nonlinear lattice problems where the resonators are assumed to be nonlinear and the bonds are taken as linear, here we assume the opposite, namely that the nonlinearity is introduced only in the coupling bonds (intracell) and that it depends on the intensity on them as $\left|a_{m, n}^{(j)}-a_{m, n}^{(j)}\right|^{2}$. As function of the intensity on the bond, the effective nonlinear coupling coefficient varies from $\kappa_{0}$ at very low intensities to $\kappa_{\infty}$ at high intensities. If the linear intercell coupling $v$ is chosen so that $\kappa_{0}<v<\kappa_{\infty}$, intensity-induced topological transitions take place, thus giving rise to selfinduced topologically protected edge-states that are immune against certain defects in the lattice. In the next sections, we study different aspects of this behavior, beginning with the topological properties of the infinite nonlinear lattice, moving to frequency domain nonlinear eigenvalue-eigenvector analysis of a finite strip and concluding with numerically exact time domain simulations of a finite nonlinear sample.

\section{B. Quasilinear dispersion analysis of the infinite lattice}

Assuming no resonances at higher harmonics of the fundamental resonance frequency $\omega_{0}$, the Manley-Rowe relations ensure, to sufficiently good extent, frequency mixing due to the nonlinearity can be neglected, and thus a time harmonic analysis of the structure is justifiable in a linearized framework. Under this assumption, the bulk dispersion of the infinite, shift-invariant structure can be found by plugging the following ansatz of time harmonic Bloch-like solutions, $a_{m, n}^{(j)}=\sqrt{I} a^{(j)} e^{i(m \theta+n \phi-\omega t)}$, into Eqs. (1). Here, $\phi=k_{x} a, \phi \in$ $[-\pi,+\pi)$, and $\theta=\left[(1 / 2) k_{x}+(\sqrt{3} / 2) k_{y}\right] a, \theta \in[-\pi,+\pi)$ are Bloch phases along the $\hat{x}$ and the $\hat{s}$ axis, respectively, $k_{x, y}$ are the wave-vector components, and $I=\sum_{j=1}^{3}\left|a_{m, n}^{(j)}\right|^{2}$ is the total intensity in the trimer. By choosing the Bloch amplitudes $a^{(j)}$ to be subject to the normalization, $\sum_{j=1}^{3}\left|a^{(j)}\right|^{2}=1$, the system of Eqs. (1) is reduced to the nonlinear eigenvalue problem $\hat{\mathcal{H}}_{\mathrm{TB}} \boldsymbol{a}=\bar{\omega} \boldsymbol{a}$, with the tight-binding Hamiltonian within each unit cell given by

$$
\hat{\mathcal{H}}_{\mathrm{TB}}=\left(\begin{array}{ccc}
0 & \kappa^{(2,1)}\left(a^{(2)}, a^{(1)}\right)+v e^{i(\theta-\phi)} & \kappa^{(3,1)}\left(a^{(3)}, a^{(1)}\right)+v e^{i \theta} \\
\kappa^{(1,2)}\left(a^{(1)}, a^{(2)}\right)+v e^{-i(\theta-\phi)} & 0 & \kappa^{(3,2)}\left(a^{(3)}, a^{(2)}\right)+v e^{i \phi} \\
\kappa^{(1,3)}\left(a^{(1)}, a^{(3)}\right)+v e^{-i \theta} & \kappa^{(2,3)}\left(a^{(2)}, a^{(3)}\right)+v e^{-i \phi} & 0
\end{array}\right),
$$

where $\bar{\omega}=\omega-\omega_{0}$ denotes the frequency detuning between the excitation frequency and the resonance frequency of a single isolated resonator, the column vector $\boldsymbol{a}=\left[a^{(1)}, a^{(2)}, a^{(3)}\right]^{T}$ denotes the relative distribution of the modal amplitudes at the $m, n=0,0$ reference trimer, and $k^{(i, j)}\left(a^{(i)}, a^{(j)}\right)=\kappa_{\infty}+\frac{\kappa_{0}-\kappa_{\infty}}{1+\alpha I\left|a^{(j)}-a^{(i)}\right|^{2}}$ is the nonlinear, amplitude-dependent, coupling coefficient on the reference trimer. For real $\kappa_{0}, \kappa_{\infty}$ and $v$, the $3 \times 3$ matrix (2) is Hermitian, and hence its eigenvalues are real.

In the linear limit $(\alpha=0)$, the respective eigenvalue problem is solved analytically. For positive $\kappa=\kappa_{0}, v>0$, the band structure features three pass bands, with two dispersive 
bands (lower band $\bar{\omega}_{-}$and upper band $\bar{\omega}_{+}$) given by

$$
\begin{aligned}
& \bar{\omega}_{ \pm}(\theta, \phi) \\
& \quad=\frac{1}{2}\{\kappa+v \pm \sqrt{\delta+8 \kappa v[\cos \theta+\cos (\theta-\phi)+\cos \phi]}\}
\end{aligned}
$$

where $\delta=9 \kappa^{2}-6 \kappa \nu+8 v^{2}$, and a lowest flat band of constant frequency $\bar{\omega}_{\text {flat }}=-(\nu+\kappa)$. At the $\Gamma$ point, i.e., $(\theta, \phi)=(0,0)$, the lower and flat bands are degenerate for any choice of the intercell and intracell coupling parameters. Near the Dirac points $\left(\theta_{D}, \phi_{D}\right)=(\mp 2 \pi / 3, \pm 2 \pi / 3)$, the dispersion relation (3) can be rewritten as $\left(\bar{\omega}_{ \pm}-\frac{\kappa+v}{2}\right)^{2}=$ $\frac{9}{4}(\kappa-v)^{2}+\frac{3}{4} \kappa v a^{2}\left(\tilde{k}_{x}^{2}+\tilde{k}_{y}^{2}\right)$, where $\tilde{k}_{x, y}$ are small deviations. At $\tilde{k}_{x}=\tilde{k}_{y}=0$, it reduces to $\bar{\omega}_{ \pm}=\frac{\kappa+v}{2} \pm \frac{3}{2}(\kappa-v)$, and thereby the spectral width of the bandgap between $\bar{\omega}_{+}=2 \kappa-v$ and $\bar{\omega}_{-}=2 v-\kappa$ is found in closed form as $\Delta \bar{\omega}_{\text {gap }} \equiv\left|\omega_{+}-\omega_{-}\right|=3|\kappa-\nu|$. For $\kappa=v$, two bands intersect $\bar{\omega}_{+}=\bar{\omega}_{-}=v$, locally exhibiting Dirac crossings $\left(\bar{\omega}_{ \pm}-v\right)^{2}=\frac{3}{4} v^{2} a^{2}\left(\tilde{k}_{x}^{2}+\tilde{k}_{y}^{2}\right)$. The linear topological properties of this system can be unveiled by calculating the generalized winding number for two bands $w_{ \pm}$, which is regarded as the generalization to the $2 \mathrm{D}$ scenario of the winding number for the 1D SSH [38]. A nonzero value of this topological invariant indicates the existence of a nontrivial gap and, alongside, the emergence of topologically protected edge states. Following Ref. [37], we obtain $w_{ \pm}= \pm \frac{1}{2}\left[1-\frac{\kappa-v}{|\kappa-v|}\right]$. Hence, at $\kappa>v$ the band structure admits a trivial gap $\left(w_{ \pm}=0\right)$, while for $\kappa<v$ the gap becomes nontrivial $\left(w_{ \pm}= \pm 1\right)$. In the nontrivial case, two edge modes per frequency emerge in the band gap. As discussed in the subsequent sections, these modes are chiral with either right-handed or left-handed circular polarization, and are topologically protected against defects that preserve their chiral symmetry, which is at the root of their topological properties.

The nonlinear response is more challenging to tackle analytically. However, in order to get some physical insights into the topological properties of the structure as a function of intensity it is enough to focus on the intensity-induced band structure dynamics at the Dirac points in the wavenumber plane $(\phi, \theta)$. The linear eigenvalues at the Dirac points are given by $\boldsymbol{a}_{\mathrm{flat}}=(1 / \sqrt{3})\left[1, e^{\frac{i 2 \pi}{3}}, e^{-\frac{i 2 \pi}{3}}\right]^{T}$ for the flat band, $\boldsymbol{a}_{-}=(1 / \sqrt{3})\left[1, e^{-\frac{i 2 \pi}{3}}, e^{\frac{i 2 \pi}{3}}\right]^{T}$ for the lower band and $\boldsymbol{a}_{+}=(1 / \sqrt{3})[1,1,1]^{T}$ for the upper band. Now, as a first approximation, we substitute the expression of the linear eigenvalues at the Dirac points into the nonlinear coupling coefficient of the reference trimer $k^{(i, j)}\left(a^{(i)}, a^{(j)}\right)$ and solve the ensuing eigenvalue problem defined by Eq. (2). For $\kappa_{0}>v>\kappa_{\infty}$, we obtain three resonance frequencies, one that corresponds to the flat band $\bar{\omega}_{\text {flat }}=-\left(\kappa_{\text {eff }}(I)+v\right)$, and additional two frequencies $\bar{\omega}_{+}$and $\bar{\omega}_{-}$that depend on the intensity, whether it is above or below the threshold intensity $I_{\text {th }}=3\left(\kappa_{0}-v\right) / \alpha\left[3 v-2 \kappa_{0}-\kappa_{\infty}\right]$ at which the gap closes. For intensities below the threshold $I<I_{\text {th }}$ we find $\bar{\omega}_{-}=2 v-\kappa_{\text {eff }}(I)$ and $\bar{\omega}_{+}=2 \kappa_{0}-v$, while for intensities above the threshold $I>I_{\text {th }}$ the solutions flip and we find $\bar{\omega}_{-}=2 \kappa_{0}-v$ and $\bar{\omega}_{+}=2 v-\kappa_{\text {eff }}(I)$. Here, $\kappa_{\text {eff }}(I)=\kappa_{\infty}+$ $\left(\kappa_{0}-\kappa_{\infty}\right) /(1+\alpha I)$ is an effective nonlinear intracell coupling dependent on the control parameter $\alpha I$.
The nonlinear bandgap dynamics at the Dirac points is illustrated in Figs. 1(b)-1(d). Figure 1(b) shows the variation of the effective coupling coefficient as function of intensity. Figure 1(c) shows the corresponding frequency solution evolution vs intensity. This result is then used to calculate the bandgap width, shown in Fig. 1(d), demonstrating that the bandgap closes and reopens at the threshold intensity $I_{\text {th }}$. We emphasize, however, that this analysis is strictly valid only in the vicinity of the Dirac point and for not-too-large intensities so that the substitution of the linear eigenvalues in the nonlinear coupling of the reference trimer is justified. The above analysis serves the purpose of providing physical insights in the nonlinear topological transition. The problem will be numerically tackled without approximations in the subsequent sections and a more refined semianalytical analysis will be given in the Appendix.

\section{Study of an infinite strip}

As pointed out in the literature [39], an effective approach to obtain topologically protected edge modes is to first identify the points in momentum space where the frequency spectrum is gapless, and then properly tune the system parameters to reopen the gap in a different topological state. The new band structure can then change topological phase, and one consequence of this is the emergence of topologically protected gap modes. Generally the points where the gap can be closed and then reopened are points of degeneracy, i.e., cusps, in the band structure. In 2D geometries, the most common points of degeneracy are Dirac points. In our geometry, the fact that at a certain threshold intensity the bandgap can close and reopen at the Dirac points as a function of applied intensity is an indication that the system topology is not preserved intact, giving rise to edge modes with topological protection. To explore these modes, we next study the wave dynamics in the vicinity of the boundaries of a strip, which is finite along the slanted axis and infinite along the other principal axis, or vice-versa. Figure 2 shows two different strip geometries that we consider. Since translational invariance is preserved along the $x(s)$ axis, the Bloch phase $\phi(\theta)$ can be used, reducing the infinite strip problem to a finite chain problem along the $s(x)$ axis. We denote two different cuts by Edge $\mathrm{e}_{j, \phi}\left(\operatorname{Edge}_{j, \theta}\right)$ with $j=1,2$. These two arrangements are equivalent under the transformations $\phi \rightarrow \theta, 2 \rightarrow 1,1 \rightarrow 2$.

The band structure of the infinite strip in Fig. 2(a) in the low intensity limit (linear lattice limit) is shown in Fig. 3(a). In this case we set the intensity $\alpha I_{\text {tot }}=0.01 \ll 1$, where $I_{\text {tot }}$ is the total intensity in the finite chain along the slanted axis (sum over the intensities in all the $N=25$ trimers). Since the strip is finite along the slanted axis, the band structure is one-dimensional with Bloch phase $\phi$ and contains $3 \times M$ dispersion bands, which should be studied in the first Brillouin zone $\phi \in[-\pi, \pi]$. Along the slanted axis, the strip is finite and the mode amplitudes are found as the elements of the eigenvectors, which are bulk modes distributed over the entire strip width. For example, Fig. 3(b) shows the eigenvector that corresponds to the frequency-wave-number point marked by the red dot in Fig. 3(a). The field distribution exhibits a typical bulk-mode cosine-like shape. For different frequencywave-number points the amplitude distribution changes, but 


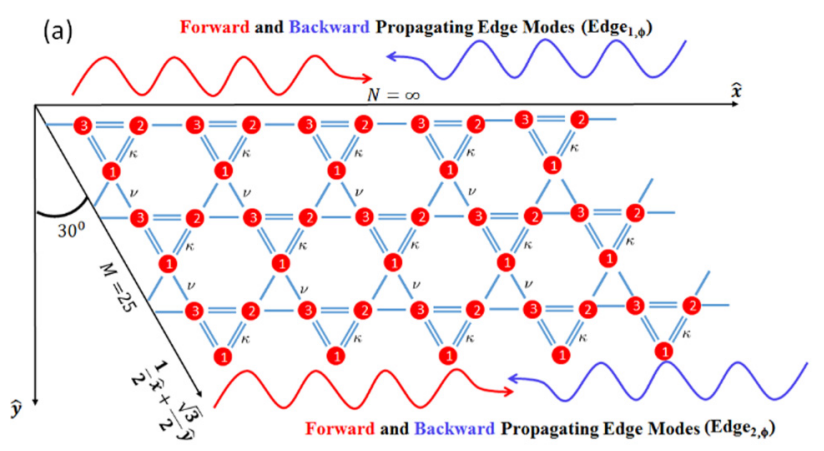

(b)

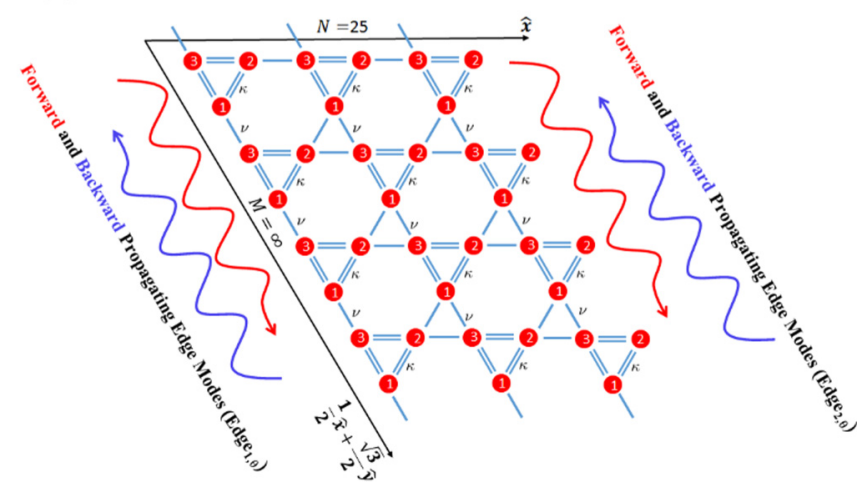

FIG. 2. Finite strip geometries made of $M=25$ trimers along the slanted axis (a), and $N=25$ trimers along the $\hat{x}$ axis (b). On both edges forward and backward waves are supported.

it always fills the entire strip width. As opposed to the low intensity limit, by increasing the intensity parameter so that $\alpha I_{\text {tot }} \gg 1$, saturation is achieved and the band structure of the lattice dramatically changes. This is shown in Fig. 3(c), where we observe that the band gap widens near the Dirac points, turning the two band edge dispersion lines into ingap edge modes. Now, the red point in the frequency-wavenumber space falls onto a topologically nontrivial edge-mode dispersion branch, and it corresponds to the field profile shown in Fig. 3(d). This field becomes localized near Edge ${ }_{1, \phi}$ and drops away from the edge along the slanted axis. However, as the local intensity declines, the band structure is locally modified, and consequently the field decay rate changes until a plateau-like level at about $\alpha I_{\text {th }}=15$ is reached. The plateau level coincides with the intensity threshold at which the bandgap is completely closed, and the lattice topology is locally at the borderline between trivial and nontrivial. The field intensity difference from the asymptotic prediction is only near the other edge $(2, \phi)$, due to edge effects. It is important to emphasize here that, while in the low and high intensity limits the structure dynamics can be described using quasilinear analysis based on band structure study, strictly speaking this kind of analysis cannot be justified in the intermediate range of intensities when $\alpha I_{\text {tot }} \sim 1$. In this range one cannot avoid a study of the full nonlinear dynamics in order to have a complete picture of the wave species supported by the structure. In this case, soliton solutions may be expected [30], possibly together with bistability and multistability effects. These features, which may arise in the range of intensities that fall between the two extreme scenarios (low and high
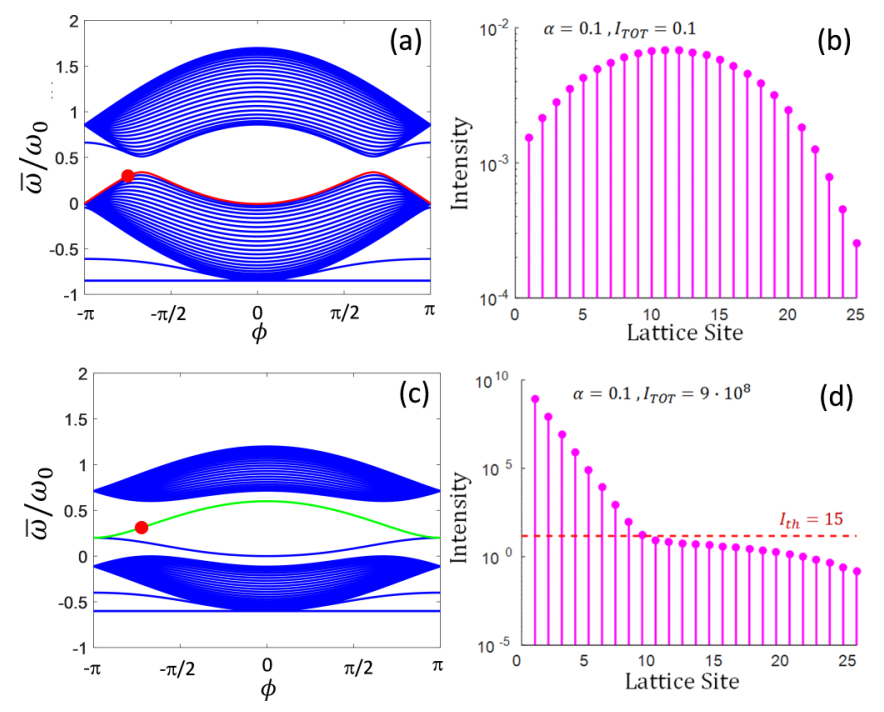

FIG. 3. (a) Band structure for the strip geometry depicted in Fig. 2(a) in the quasilinear regime, $I_{\text {tot }}=0.1, \alpha=0.1$. The parameters used for the saturable nonlinearity are: $\kappa_{\infty}=0.2 \omega_{0}, \kappa=$ $0.45 \omega_{0}$, and $v=0.4 \omega_{0}$. (b) Bulk-mode field distribution calculated for the point marked by the red dot in panel (a). This point located at the lower-frequency band-edge dispersion line is characterized by $\bar{\omega}=0.3 \omega_{0}$ and the backward momentum $\phi=-2.33$. (c) Band structure in the nonlinear case at $\alpha I_{\text {tot }} \gg 1$, at $\alpha=0.1$ and $I_{\text {tot }}=$ $9 \times 10^{8}$. The red dot now falls onto the topological edge-mode dispersion line. (d) Field localization of the edge mode. The field localization tends to the threshold value $I_{\text {th }}=15$ predicted by the Bloch analysis of the infinite lattice.

intensities) on which we focus this paper, are not considered further. Finally, the results for the case of Fig. 2(b) coincide with those described above, under the transformation $\phi \rightarrow \theta$, $a_{2} \rightarrow a_{1}, a_{1} \rightarrow a_{2}$.

\section{Chirality of the edge modes}

The chiral nature of the edge modes is essential to define and support their topological properties. Based on their chirality, it is possible to give an under-the-hood explanation for the topological protection of the in-gap edge modes against certain types of defects. We calculate the chirality for both forward and backward-propagating edge modes along the four edges by expanding the fields over any trimer in the basis

$$
\left[\begin{array}{l}
a_{1} \\
a_{2} \\
a_{3}
\end{array}\right]=\frac{C_{\mathrm{CM}}}{\sqrt{3}}\left[\begin{array}{l}
1 \\
1 \\
1
\end{array}\right]+\frac{C_{+}}{\sqrt{3}}\left[\begin{array}{c}
1 \\
e^{\frac{i 2 \pi}{3}} \\
e^{\frac{i 4 \pi}{3}}
\end{array}\right]+\frac{C_{-}}{\sqrt{3}}\left[\begin{array}{c}
1 \\
e^{-\frac{i 2 \pi}{3}} \\
e^{-\frac{i 4 \pi}{3}}
\end{array}\right],
$$

where $C_{+}$and $C_{-}$are the amplitudes of the left-handed and right-handed polarized modes, respectively, and $C_{\mathrm{CM}}$ is the amplitude of the common mode. The chirality $\chi=\frac{\left|C_{+}\right|-\left|C_{-}\right|}{\left|C_{+}\right|+\left|C_{-}\right|}$. We find that at the corner between Edge $_{1, \phi}$ and Edge $_{1, \theta}$ chirality is conserved between the backward propagating edge mode $(\phi<0)$ along the $\hat{x}$ axis and the forward propagating edge mode $(\theta>0)$ along the $\hat{s}$ axis, and, vice versa, between the forward propagating edge mode $(\phi>0)$ along the $\hat{x}$ axis and the backward propagating edge mode $(\theta<0)$ along the 


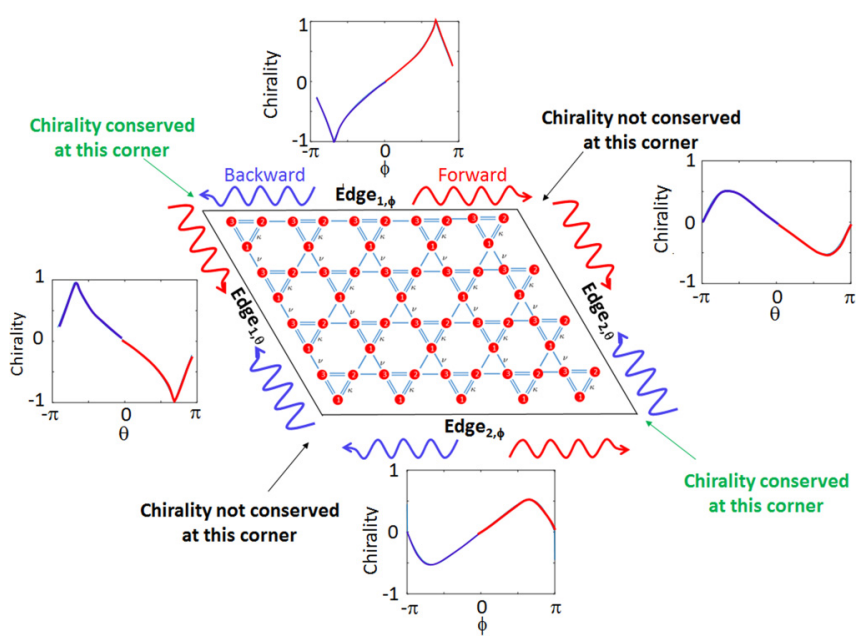

FIG. 4. Chirality conservation properties along the four edges and at the different corner types.

$\hat{s}$ axis. Likewise, chirality does not change at the corner between Edge $e_{2, \phi}$ and Edge ${ }_{2, \theta}$, while it is never conserved at the corner between Edge $_{1,(2), \phi}$ and Edge ${ }_{2,(1), \theta}$. Importantly, chirality conservation generally implies zero scattering of an edge mode upon interaction with the corner, whereas in the case of chiral mixing back scattering should be expected. The results of this study are summarized in Fig. 4: a defect at the edge that preserves the chirality of the edge mode will not create scattering in the bulk, since the edge mode is in the gap, whereas it will also not create reflection to an edge mode that propagate to the opposite direction, due to the conservation of chirality.

\section{E. Spatiotemporal dynamics in finite arrays with defects}

Up to now our analysis involved a frequency-domain analysis based on a quasilinear approach. In this section, we verify these results and conclusions based on a numerically exact time-domain simulation of the system of equations (1) for a finite Kagome lattice with defects. The lattice contains 20 trimers along $m$ and 25 along $n$, as shown in Fig. 5. All trimers in the lattice, except for those in the defected region, are identical and characterized by the same self-resonance frequency, and linear and nonlinear coupling coefficients. The defected region is the rhombus area in the upper left corner of the lattice, with resonators depicted by red dots. In this region, the self-frequency of each resonator is set to be zero.

The lattice is excited through a wave port that is coupled to resonator number 3 in the trimer at the upper-right corner of the lattice, namely, to resonator $a_{1,25}^{(3)}$. The input port point is specified in Fig. 5 by the red arrow. To have a source that can achieve steady state, we modify the equation describing the time evolution of resonator $a_{1,25}^{(3)}$ as follows. First, we add a source coupling term $i d S_{\text {in }}$, where $S_{\text {in }}=S_{0} e^{-i \omega t}$, with $\bar{\omega}=$ $\omega-\omega_{0}=0.3 \omega_{0}$ and $d$ being the input coupling coefficient. Second, we set the self-resonance frequency of this resonator to be complex rather than real, $\omega_{0} \rightarrow \omega_{0}-i \gamma$, to model the effect of back reflection from the port. We found numerically

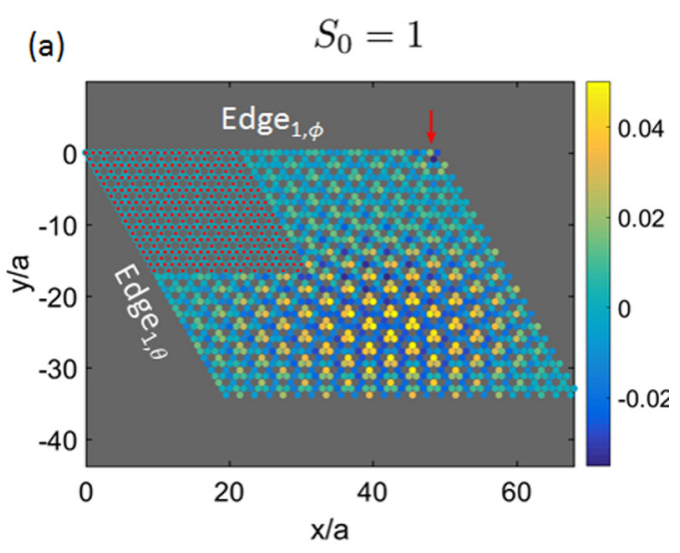

(b)

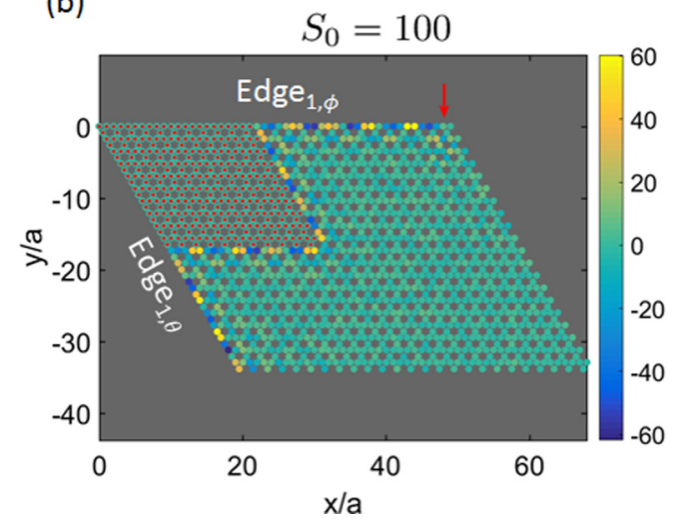

FIG. 5. Self-induced topological transition and protection in a finite-size lattice with defects. The defective resonators are delineated with red dots. The real part of the mode amplitude $\operatorname{Re}\{a\}$ is color coded. The simulation parameters, as before, are $\kappa_{\infty}=$ $0.2 \omega_{0}, \quad \kappa_{0}=0.45 \omega_{0}, \quad v=0.4 \omega_{0}, \alpha=0.1$. (a) At low input-port intensity $S_{0}=1$ a bulk mode profile is excited, being affected by the defect region shape. (b) At the input intensity $S_{0}=100$, the lattice topology is nontrivial, giving rise to reflection-less propagation of the localized edge mode despite the corners (see Supplemental Material Ref. [40]).

that $\gamma=0.092 \omega_{0}$ yields impedance matching, with optimal coupling efficiency and minimal reflection. Additionally, energy conservation requires $d=\sqrt{2 \gamma}$. Therefore, the reflected field reads $S_{\text {out }}=-S_{\text {in }}+d a_{1,25}^{(3)}$.

Once the structure is excited, we let the fields evolve for long enough time and then we take a snapshot capturing the instantaneous fields $a_{m, n}^{(i)}(t)$ at each resonator in the lattice. Figure 5(a) shows the excitation of a bulk mode in the lattice at low input intensity $S_{0}=1$. As discussed above, this is the only type of mode supported in the quasilinear topologically trivial structure. However, the bulk mode is seen to be significantly altered by the corners present in the lattice because of the defect area. The dynamics drastically changes as we increase the input port intensity by two orders of magnitude to $S_{0}=100$. Here, we observe propagation of the wave localized to the edge. This wave experiences no reflections despite the presence of several corners, manifesting chirality conservation. In this case, the lattice topology has experienced a self-induced transition to become nontrivial. 


\section{CONCLUSIONS}

In this paper, we have demonstrated the emergence and routing of topologically nontrivial edge states of pure nonlinear origin in a two-dimensional metasurface supporting an intensity-induced topological transition. Given that nonlinear interactions are ubiquitous and our model based on nonlinearly coupled oscillators is applicable to a variety of physical systems, there are expectations for experimental observations of the predicted effects in a wide range of metasurfaces, including optical, electronic, acoustic, and mechanical structures. An experimental demonstration of nonlinearityinduced corner modes in a different circuit platform has been recently presented [41]. The different symmetry in the considered array in Ref. [41] would not support the emergence of nonlinearity-induced topological edge modes, but it does sustain higher-order topological states pinned to the corners. Our work opens several opportunities to experimentally realize topological transitions, self-induced topological edge propagation in a variety of platforms. In electronic circuits, for instance, generalizing the platform introduced in Ref. [29] to two dimensions, it may be possible to implement selfreconfigured multiplexed lines with topological robustness. In radio frequencies and in optics, it may be possible to observe these effects using nonlinear metasurfaces. Of particular interest would be to explore the unusual dynamics of these nonlinear systems in the range of intensities around the topological transition, not analyzed in the present work. We expect the insurgence of bistability or multistability regimes, and the excitation of topological solitons associated with moving domain walls, as analyzed in the 1D scenario in Ref. [30]. These opporunities pave the way for future advances in the implementation of robust wave transport based on nontrivial topological states.

\section{ACKNOWLEDGMENT}

This work has been supported by the Office of Naval Research, the Air Force Office of Scientific Research, and the Simons Foundation.

\section{APPENDIX: NEAR DIRAC-POINT ANALYSIS}

To gain a better understanding of the simulated topological transition, we have developed a semianalytical model for the nonlinearity-induced topological edge states. We first refer to the linear implementation of Hamiltonian (2) obtained within the tight-binding model [37]. We then perform the transformation $\hat{\mathcal{H}}=U \hat{\mathcal{H}}_{\mathrm{TB}} U^{-1}$ with the matrix

$$
U=\frac{1}{\sqrt{3}}\left(\begin{array}{ccc}
1 & 1 & 1 \\
1 & e^{-\frac{2 i \pi}{3}} & e^{\frac{2 i \pi}{3}} \\
1 & e^{\frac{2 i \pi}{3}} & e^{-\frac{2 i \pi}{3}}
\end{array}\right)
$$

Note that the matrix $U$ in Eq. (A1) is composed of the common monopolar mode as well as of the right- and lefthand circular modes introduced in Sec. II B to study the chiral phenomena. The Hamiltonian expanded near $K\left(K^{\prime}\right)$ up to first-order in wave number $\tilde{\boldsymbol{k}}$ is recast to

$$
\hat{\mathcal{H}}=\left(\begin{array}{cc}
\frac{\kappa+v}{2}+\frac{3}{2}(\kappa-v) & \frac{\sqrt{3}}{2} \nu a\left( \pm \tilde{k}_{x}+i \tilde{k}_{y}\right) \\
\frac{\sqrt{3}}{2} \nu a\left( \pm \tilde{k}_{x}-i \tilde{k}_{y}\right) & \frac{\kappa+v}{2}-\frac{3}{2}(\kappa-v)
\end{array}\right),
$$

where we denote the effective mass term $m=3(\kappa-v) / 2$, the midgap frequency $\bar{\omega}_{0}=(\kappa+v) / 2$ and the Dirac velocity $V=\sqrt{3} v a / 2$. Here, we assume a nearly degenerate scenario $\kappa \approx v$, changing the basis to the two-component vector $\left[\psi_{1}, \psi_{2}\right]^{T}$ associated with the monopolar and circularly polarized dipolar modes supported by the trimer, and the second dipolar mode is eliminated as being remote in frequency from the degeneracy point [37].

The previous analysis [37] suggests that the linear case with $\kappa<v$, or equivalently $m<0$, is nontrivial and an edge state crossing the gap arises. Considering the boundary of the nontrivial crystal $(y>0)$ and vacuum, we apply two consistent approaches to calculate the dispersion branches of edge modes (the $\left(a_{2}, a_{3}\right)$ cut, termed Edge $1, \phi$ above, is considered here): (i) numerical, using the three-site tight-binding model for the cut Kagome lattice, and (ii) analytical, exploiting the reduced two-band model. In the latter instance, we accept the ansatz $\sim \exp \left(i \tilde{k}_{x} x-\varkappa y\right)$ for the edge-bound solution and
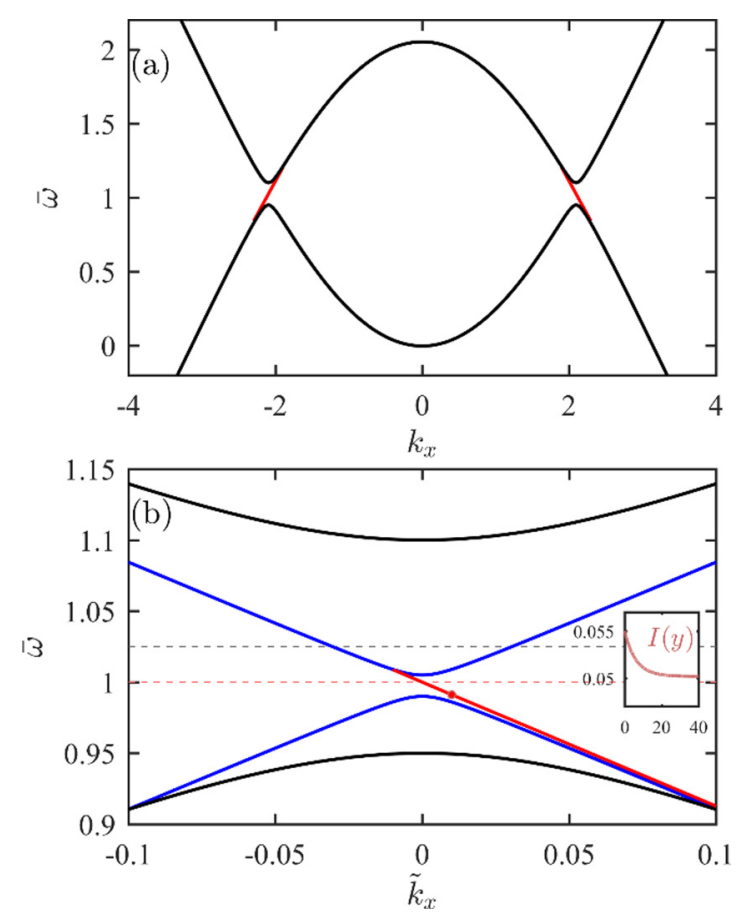

FIG. 6. (a) Band structure of the linear nontrivial Kagome lattice (two upper bands) and branches of the edge states residing in different valleys. Parameters are $\kappa=1, v=1.05$. (b) Vicinity of the Dirac point: gapped linear bulk dispersion (solid black curves), nonlinear bulk dispersion (solid blue) in the infinite lattice at the intensity $I=1.1 I_{\text {th }}$ with the inverted topological bands, and edge state dispersion calculated at $I(0)=1.1 I_{\text {th }}$ (solid red). Dashed gray and red lines mark the frequencies $\bar{\omega}=(\tilde{\kappa}+v) / 2$ and $\bar{\omega}=v$, respectively. The inset shows the edge state profile $I(y)$ calculated at wave number $\tilde{k}_{x}=0.01$, specified by the red point. Parameters are $\tilde{\kappa}=1.05, v=1, \tilde{\alpha}=1$. The edge solution decays to the plateau level $I_{\mathrm{th}}=(\tilde{\kappa}-v) / \tilde{\alpha}=0.05$. 
formally utilize the effective boundary condition of the form $\psi_{2}(0)=-\psi_{1}(0)$ at $y=0$, leading to

$$
\frac{\psi_{1}(0)}{\psi_{2}(0)}=\frac{V\left( \pm \tilde{k}_{x}-\varkappa\right)}{\Omega-m}=-1
$$

where the decay factor $\varkappa=\sqrt{V^{2} \tilde{k}_{x}^{2}+m^{2}-\Omega^{2}} / V$, and $\Omega=$ $\bar{\omega}-\bar{\omega}_{0}$ is a deviation from the midgap frequency. By both methods, the dispersion of the edge state near the Diract point is found to be linear, $\Omega=\mp V \tilde{k}_{x}$ (opposite signs in different valleys), see Fig. 6(a). Remarkably, Eq. (A3) can be satisfied only if $|m|=-m$, i.e., for negative masses. The applicability of the effective boundary condition is confirmed by the transformation $\left[\psi_{1}(0), \psi_{2}(0), 0\right]^{T}=U\left[a_{1}, a_{2}, a_{3}\right]_{y=0}^{T}$, where $\left[a_{1}, a_{2}, a_{3}\right]_{y=0}^{T}$ is the amplitude vector in the trimer at the boundary row calculated numerically, with $a_{2}=$ $\left|a_{2}\right| \exp \left(\frac{i \pi}{6}\right)=a_{3}^{*},\left|a_{1}\right| \approx 0$. Therefore, this boundary condition is employed in the following nonlinear generalization of the two-band model.
We assume almost equal bonds in-between the cavities of the trimer, tracing back our analysis to the case of nonlinear intracell coupling $\kappa(I)$ of nonlocal nature uniform over the unit cell. Following the procedure outlined in Ref. [37], but extended here to the nonlinear problem, we find the expression for the winding number in the nonlinear case $w_{ \pm}= \pm \frac{1}{2}\left[1-\frac{\kappa(I)-v}{|\kappa(I)-v|}\right]$, and deduce that the band structure admits a trivial gap $\left(w_{ \pm}=0\right)$ for $I<I_{\mathrm{th}}$, where the threshold intensity $I_{\text {th }}$ is determined from the condition $\kappa\left(I_{\text {th }}\right)=v$. The gap becomes nontrivial $\left(w_{ \pm}= \pm 1\right)$ for $I>I_{\mathrm{th}}$, implying the appearance of protected edge modes.

We examine closer the nonlinear case of intensitydependent intracell coupling $\kappa(I)=\tilde{\kappa}-\tilde{\alpha} I$, where $\tilde{\kappa}, \tilde{\alpha}>0$ are coefficients that are used to approximate the saturable nonlinear model we originally consider, and the total intensity in the trimer is expressed by $I=\left|\psi_{1}\right|^{2}+\left|\psi_{2}\right|^{2}$. Here, we utilize a simplified formula for $\kappa(I)$, given the weak nonlinearity sufficient to close the gap in the nearly degenerate case $\kappa \gtrsim \nu$. Accordingly, the coupled-mode equations are modified as

$$
\left(\begin{array}{cc}
2 \tilde{\kappa}-v-2 \tilde{\alpha}\left(\left|\psi_{1}\right|^{2}+\left|\psi_{2}\right|^{2}\right)-\bar{\omega} & V\left(\tilde{k}_{x}+i \tilde{k}_{y}\right) \\
V\left(\tilde{k}_{x}-i \tilde{k}_{y}\right) & 2 v-\tilde{\kappa}+\tilde{\alpha}\left(\left|\psi_{1}\right|^{2}+\left|\psi_{2}\right|^{2}\right)-\bar{\omega}
\end{array}\right)\left(\begin{array}{l}
\psi_{1} \\
\psi_{2}
\end{array}\right)=0
$$

System (A4) comprises two coupled nonlinear ordinary differential equations,

$$
\begin{aligned}
& \frac{d \psi_{2}}{d y}=-V \tilde{k}_{x} \psi_{2}-\left(2 \tilde{\kappa}-v-\bar{\omega}-2 \tilde{\alpha}\left[\left|\psi_{1}\right|^{2}+\left|\psi_{2}\right|^{2}\right]\right) \psi_{1}, \\
& \frac{d \psi_{1}}{d y}=V \tilde{k}_{x} \psi_{1}+\left(2 v-\tilde{\kappa}-\bar{\omega}+\tilde{\alpha}\left[\left|\psi_{1}\right|^{2}+\left|\psi_{2}\right|^{2}\right]\right) \psi_{2} .
\end{aligned}
$$

Here we can assume $\psi_{1,2}$ real. Condition $\partial_{y}=0$ in Eqs. (A5) yields the steady states $\psi_{1,2}\left(\omega, \tilde{k}_{x}\right)$ or the nonlinear dispersion $\bar{\omega}\left(\tilde{k}_{x}, I\right)$ in infinite arrays, which reveals that for $\kappa>v$ with increasing intensity, the bandgap gradually closes and reopens. At the threshold intensity defined as $\tilde{\alpha} I_{\text {th }}=\kappa-v$, the bulk dispersion is gapless and linear $\bar{\omega}=v \pm V \tilde{k}_{x}$.
Next, we look for the edge state solutions $\psi_{1,2}(y) e^{i \tilde{k}_{x} x}$ bound to the interface $y=0$ with boundary condition $\psi_{2}(0)=$ $-\psi_{1}(0)$ imposed. Taking $\tilde{k}_{x}=0$, from Eqs. (A5) we find that an edge state arises in the topological bandgap at frequency $\bar{\omega}=v$ for interface intensities larger than the threshold value, $I(y=0)>I_{\text {th }}$. In contrast to the linear case, the decay in the bulk sits on the steady-state background $\left|\psi_{1}\right|^{2}+\left|\psi_{2}\right|^{2}=$ $(\tilde{\kappa}-v) / \tilde{\alpha}$. For nonzero wave vectors $\tilde{k}_{x}$, the edge wave decays asymptotically to the same plateau level away from the boundary.

The dispersion of the nonlinear topological edge state is found by solving Eqs. (A4) at the above-threshold intensity $I(0)$, see Fig. 6(b). No solutions of this type can be obtained in the quasilinear trivial case. It is clear that this nonlinearlyinduced band inversion, and corresponding topological transition, is responsible for the emergence of the topological edge modes.
[1] M. Z. Hasan and C. L. Kane, Colloquium: Topological insulators, Rev. Mod. Phys. 82, 3045 (2010).

[2] X.-L. Qi and S.-C. Zhang, Topological insulators and superconductors, Rev. Mod. Phys. 83, 1057 (2011).

[3] C. L. Kane and E. J. Mele, $Z_{2}$ Topological Order and the Quantum Spin Hall Effect, Phys. Rev. Lett. 95, 146802 (2005).

[4] C. L. Kane and E. J. Mele, Quantum Spin Hall Effect in Graphene, Phys. Rev. Lett. 95, 226801 (2005).

[5] B. A. Bernevig, T. L. Hughes, and S.-C. Zhang, Quantum spin Hall effect and topological phase transition in HgTe quantum wells, Science 314, 1757 (2006).
[6] L. Fu, C. L. Kane, and E. J. Mele, Topological Insulators in Three Dimensions, Phys. Rev. Lett. 98, 106803 (2007).

[7] D. Hsieh, D. Qian, L. Wray, Y. Xia, Y. S. Hor, R. J. Cava, and M. Z. Hasan, A topological Dirac insulator in a quantum spin Hall phase, Nature (London) 452, 970 (2008).

[8] J. E. Moore and L. Balents, Topological invariants of timereversal-invariant band structures, Phys. Rev. B 75, 121306(R) (2007).

[9] R. Roy, $Z_{2}$ classification of quantum spin Hall systems: An approach using time-reversal invariance, Phys. Rev. B 79, 195321 (2009). 
[10] H. Zhang, C.-X. Liu, X.-L. Qi, X. Dai, Z. Fang, and S.-C. Zhang, Topological insulators in $\mathrm{Bi}_{2} \mathrm{Se}_{3}, \mathrm{Bi}_{2} \mathrm{Te}_{3}, \mathrm{Sb}_{2} \mathrm{Te}_{3}$ with a single Dirac cone on the surface, Nat. Phys. 5, 438 (2009).

[11] F. D. M. Haldane and S. Raghu, Possible Realization of Directional Optical Waveguides in Photonic Crystals with Broken Time-Reversal Symmetry, Phys. Rev. Lett. 100, 013904 (2008).

[12] Z. Wang, Y. Chong, J. D. Joannopoulos, and M. Soljačić, Observation of unidirectional backscattering-immune topological electromagnetic states, Nature (London) 461, 772 (2009).

[13] M. Hafezi, S. Mittal, J. Fan, A. Migdall, and J. M. Taylor, Imaging topological edge states in silicon photonics, Nat. Photonics 7, 1001 (2013).

[14] M. C. Rechtsman, J. M. Zeuner, Y. Plotnik, Y. Lumer, D. Podolsky, F. Dreisow, S. Nolte, M. Segev, and A. Szameit, Photonic floquet topological insulators, Nature (London) 496, 196 (2013).

[15] A. B. Khanikaev, S. H. Mousavi, W. K. Tse, M. Kargarian, A. H. MacDonald, and G. Shvets, Photonic topological insulators, Nat. Mater 12, 233 (2013).

[16] Z. Yang, F. Gao, X. Shi, X. Lin, Z. Gao, Y. Chong, and B. Zhang, Topological Acoustics, Phys. Rev. Lett. 114, 114301 (2015).

[17] A. B. Khanikaev, R. Fleury, S. H. Mousavi, and A. Alù, Topologically robust sound propagation in an angular-momentumbiased graphene-like resonator lattice, Nat. Commun. 6, 8260 (2015).

[18] R. Susstrunk and S. D. Huber, Observation of phononic helical edge states in a mechanical topological insulator, Science $\mathbf{3 4 9}$, 47 (2015).

[19] L. M. Nasha, D. Klecknera, A. Reada, V. Vitellib, A. M. Turnerc, and W. T. M. Irvine, Topological mechanics of gyroscopic metamaterials, PNAS 112, 14495 (2015).

[20] S. H. Mousavi, A. B. Khanikaev, and Z. Wang, Topologically protected elastic waves in phononic metamaterials, Nat. Commun. 6, 8682 (2015).

[21] W. P. Su, J. R. Schrieffer, and A. J. Heeger, Solitons in Polyacetylene, Phys. Rev. Lett. 42, 1698 (1979).

[22] H. Takayama, Y. R. Lin-Liu, and K. Maki, Continuum model for solitons in polyacetylene, Phys. Rev. B 21, 2388 (1980).

[23] W. P. Su, J. R. Schrieffer, and A. J. Heeger, Soliton excitations in polyacetylene, Phys. Rev. B 22, 2099 (1980).

[24] R. Jackiw and C. Rebbi, Solitons with fermion number 1/2, Phys. Rev. D 13, 3398 (1976).
[25] A. J. Heeger, S. Kiverson, J. R. Schrieffer, and W. P. Su, Solitons in conducting polymer, Rev. Mod. Phys. 60, 781 (1988).

[26] J. Ruostekoski, G. V. Dunne, and J. Javanainen, Particle Number Fractionalization of an Atomic Fermi-Dirac Gas in an Optical Lattice, Phys. Rev. Lett. 88, 180401 (2002).

[27] L. Li, Z. Xu, and S. Chen, Topological phases of generalized Su-Schrieffer-Heeger models, Phys. Rev. B 89, 085111 (2014).

[28] Y. Hadad, A. Khanikaev, and A. Alù, Self-induced topological transitions and edge states supported by nonlinear staggered potentials, Phys. Rev. B 93, 155112 (2016).

[29] Y. Hadad, J. C. Soric, A. Khanikaev, and A. Alù, Selfinduced topological protection in nonlinear circuit arrays, Nature (London) Electronics 1, 178 (2018).

[30] Y. Hadad, V. Vitelli, and A. Alù, Solitons and propagating domain-walls in topological resonator arrays, ACS Photon. 4, 1974 (2017).

[31] D. Leykam and Y.D. Chong, Edge Solitons in NonlinearPhotonic Topological Insulators, Phys. Rev. Lett. 117, 143901 (2016).

[32] O. R. Bilal, A. Foehr, and C. Daraio, Reprogrammable phononic metasurfaces, Adv. Mat. 29, 1700628 (2017).

[33] R. K. Pal, J. Vila, M. Leamy, and M. Ruzzene, Amplitudedependent topological edge states in nonlinear phononic lattices, Phys. Rev. E 97, 032209 (2018).

[34] R. Chaunsali and G. Theocharis, Self-induced topological transition in phononic crystals by nonlinearity management, Phys. Rev. B 100, 014302 (2019).

[35] H.-M. Guo and M. Franz, Topological insulator on the kagome lattice, Phys. Rev. B 80, 113102 (2009).

[36] C. H. Redder and G. S. Uhrig, Topologically nontrivial Hofstadter bands on the kagome lattice, Phys. Rev. A 93, 033654 (2016).

[37] X. Ni, M. A. Gorlach, A. Alù, and A. B. Khanikaev, Topological edge states in acoustic Kagome lattices, New J. Phys. 19, 055002 (2017).

[38] J. K. Asboth, L. Oroszlány, and A. Pályi, A Short Course on Topological Insulators, Lecture Notes in Physics Series, $1^{\text {st }}$ ed. (Springer, Berlin, 2016).

[39] L. Lu, J. D. Joannopoulos, and M. Soljacic, Topological Photonics, Nat. Phot. 8, 821 (2014), and references therein.

[40] See Supplemental Material at http://link.aps.org/supplemental/ 10.1103/PhysRevB.100.214310 for animations of the excitation process for the photonic crystal in Fig. 5.

[41] F. Zangeneh-Nejad and R. Fleury, Nonlinear Second-Order Topological Insulators, Phys. Rev. Lett. 123, 053902 (2019). 